\title{
Revista Brasileira de Enfermagem REBEn \\ Infecção hospitalar: estudo de prevalência em um hospital público de ensino
}

\author{
Nosocomial infection: study of prevalence at a public teaching hospital \\ Infección hospitalaria: estúdio de prevaléncia en un hospital público y de enseñanza
}

\section{Maria Eliete Batista Moura}

Doutora em Enfermagem. Coordenadora de Pesquisa e Pós-Graduação da Faculdade NOVAFAPI, Teresina, Pl. Docente da Graduação e do Mestrado em Enfermagem da Universidade Federal do Piauí - UFPI. posgraduacao@novafapi.com.br

Sônia Maria de Araújo Campelo

Enfermeira Especialista em MédicoCirurgica. Docente da Graduação de Enfermagem da NOVAFAPI, Teresina, PI. Mestranda em Enfermagem pela UFPI. scampelo@novafapi.com.br

Francisca Cortez Prado de Brito

Enfermeira especialista em Gestão de sistemas e serviço de saúde, Gerente de Enfermagem do Hospital Getúlio Vargas, Teresina, PI, Mestranda em Enfermagem pela UFPI. cortezbrito@superig.com.br

\section{Odinéa Maria Amorim Batista}

Enfermeira especialista em Controle de Infecção Hospitalar. Docente da graduação em Enfermagem da NOVAFAPI, Teresina,

$P$ I. Mestranda em Enfermagem pela UFPI. obatista@novafapi.com.br

Telma Maria Evangelista de Araújo

Enfermeira. Doutora em Enfermagem. Professora Adjunto da UFPI, Teresina, PI e NOVAFAPI. Membro do Núcleo de Estudos em Saúde Pública NESP/UFPI. telmalys@yahoo.com.br

Adélia Dalva da Silva Oliveira

Mestre em Políticas Públicas. Docente da Faculdade NOVAFAPI, Teresina, PI. oliveiracairo@ig.com.br

\section{RESUMO}

Este estudo objetivou determinar a prevalência de infecção hospitalar $(\mathrm{HH})$ e distribuição por topografia e por microorganismo e suas sensibilidades antimicrobianas. Realizado em duas Unidades de Terapia Intensiva (UTIS) de um hospital público de ensino de Teresina, com amostragem de 394 casos de IH e processados pelo Software SPSS. A infecção respiratória foi a maior prevalência $(61,26 \%)$ na UTI Geral. Evidenciou-se maior sensibilidade bacteriana à amicacina $(52,48)$ na UTI do SPS. 0 microorganismo com maior prevalência foi a Klebsiella pneumonia $(35,46 \%)$ na UTI geral. Conclui-se que a prevalência de IH nas duas UTIs foi de $60,8 \%$, ultrapassando $45,3 \%$ do índice geral registrado nos hospitais brasileiros que é $15,5 \%$, contribuindo para aumentar a morbi - mortalidade causadas por infecções. Descritores: Enfermagem; Infecção Hospitalar; Prevalência.

\section{ABSTRACT}

This study aimed to determine the prevalence of nosocomial infection (NI) and distribution for topography and microorganism and its antimicrobial sensibility. The study was carried out in two Intensive Care Units (ICUs) of a public teaching hospital of Teresina, with sampling of $394 \mathrm{NI}$ cases and processed by the SPSS Software. The respiratory infection was the biggest prevalence $(61.26 \%)$ in the General ICU. It was found a bigger bacterial sensibility to amicacine $(52.48 \%)$ in the ICU of the ER. The microorganism with bigger prevalence was Klebsiella pneumonia (35.46\%) in the general ICU. In conclusion, the prevalence of $\mathrm{NI}$ in the two ICUs was $60.8 \%$, exceeding $45.3 \%$ of the general rate registered in the Brazilian hospitals that is $15.5 \%$, contributing to increase the morbi-mortality caused by infections. Descriptors: Nursing; Nosocomial Infection; Prevalence.

\section{RESUMEN}

Este estudio tiene como objetivo determinar la superioridad de infección hospitalaria (IH) y distribución por topografía, por microorganismos y sus sensibilidades antimicrobianas. Realizado en dos Unidades de Terapia Intensiva (UTIS) de un hospital público de enseñanza de Teresina, con muestra de 394 casos de IH y procesados con Software SPSS. La infección respiratória mostró mayor superioridad (61,26\%) en la UTI General. Evidenció mayor sensibilidad bacteriana al amicacina $(52,48 \%)$ en la UTI del SPS. El microorganismo con mayor superioridad fue Klebsiella neumonia $(35,46 \%)$ en la UTI general. Como concluye, la superioridad de IH en las dos UTIS fue 60,8\%, sobrepasando 45,3\% del índice general registrado en hospitales brasileños que es $15,5 \%$, contribuyendo para aumentar a morbi - mortalidad causadas por infecciones.

Descriptores: Enfermería; Infección Hospitalária; Prevaléncia.

Moura MEB, Campelo SMA, Brito FCP, Batista OMA, Araújo TME, Oliveira ADS. Infecção hospitalar: estudo da prevalêcia em um hospital público de ensino. Rev Bras Enferm 2007 jul-ago; 60(4):416-21.

\section{INTRODUÇÃO}

Pacientes internados em instituições de saúde estão expostos a uma ampla variedade de microorganismos patogênicos, principalmente em Unidade de Terapia Intensiva (UTI), onde o uso de antimicrobianos potentes e de largo espectro é a regra e os procedimentos invasivos é rotina. Nessa perspectiva, esta pesquisa tem como objeto de estudo a prevalência de infecções hospitalares $(\mathrm{IH})$ nas unidades de terapia intensiva de um hospital público e de ensino.

O Ministério da Saúde (MS), na Portaria n² 2.616 de 12/05/1998, define IH como a infecção adquirida após a admissão do paciente na unidade hospitalar e que se manifesta durante a internação ou após a alta, quando puder ser relacionada com a internação ou procedimentos hospitalares ${ }^{(1)}$. 
A problemática da $\mathrm{IH}$ no Brasil cresce a cada dia, considerando que 0 custo do tratamento dos clientes com IH é três vezes maior que o custo dos clientes sem infecção. Mesmo com a legislação vigente no país, os índices de $\mathrm{IH}$ permanecem altos, 15,5\%, o que corresponde a 1,18 episódios de infecção por cliente internado com IH nos hospitais brasileiros. Além disso, considera-se mais um agravante, o fato das instituições de saúde pública possuírem a maior taxa de prevalência de $\mathrm{IH}$ no país, $18,4 \%{ }^{(2)}$.

Em 2000, foi divulgada a taxa de prevalência de $I H$ dos hospitais de referência e de ensino, localizados na capital Teresina, e apresenta-se como segue: Hospital Areolino de Abreu, 37,7\%; Maternidade Dona Evangelina Rosa, 11,3\%; Hospital Infantil Lucídio Portela, 35,7\%; Hospital de Doenças Infecto-Contagiosas, $23,7 \%$, e o Hospital Getúlio Vargas, que funciona há 59 anos, possui quatrocentos e vinte e nove leitos, tem Comissão de Controle de Infecção Hospitalar (CCIH) e Serviço de Controle de Infecção Hospitalar $(\mathrm{SClH})$ estruturado, possui uma taxa de prevalência de $\mathrm{IH}$ de $31,1 \%$. Dessa forma, a prevalência de $\mathrm{IH}$ nos cinco hospitais de referência de Teresina foi de $27,9 \%{ }^{(3)}$, ou seja, $12,4 \%$ a mais do que a prevalência registrada em nível nacional que é de $15,5 \%$ (2).

Assim, para melhorar as condições de atendimento dos hospitais do Estado do Piaui em termos de controle de infecção, foi criada em 1994 a Comissão Estadual de Controle de Infecção Hospitalar (CECIH), oficializada através de Portaria ${ }^{(4)}$ e publicação no Diário Oficial da União.

Junto a CECIH foi criado o núcleo executivo do Estado, formado por técnicos (enfermeiros e médicos) com experiência em controle de infecção. Esse núcleo desenvolve um plano de controle de infecção hospitalar $(\mathrm{PCIH})$ que tem como meta a criação de $\mathrm{CClH}$ em todos os hospitais da capital e nas regionais de saúde, para desenvolverem um conjunto de ações deliberadas e sistemáticas, com vistas à redução máxima da incidência e gravidade das infecções hospitalares.

Dessa forma, todos os profissionais da saúde e demais profissionais inseridos na estrutura da assistência hospitalar, de alguma maneira, estão permanentemente participando do trabalho em serviço, do trabalho em saúde como um todo e também são responsáveis pela estrutura, processo e resultados dos Programas de Prevenção e Controle de Infecção Hospitalar.

Neste sentido, a Epidemiologia, que é uma especialidade de Saúde Coletiva e estuda a distribuição das doenças e agravos à saúde nas populações e o seu determinante, considera que o conhecimento da ocorrência e dos determinantes das doenças e agravos à saúde objetiva ações de prevenção $0^{(5)}$. Diante disso é que os estudos epidemiológicos na área de Infecção Hospitalar têm-se destacado e se tornado mais sofisticado nos últimos anos. Dentre esses estudos, os de prevalência de $\mathrm{IH}$ medem o status de uma população com relação ao evento ou doença de interesse.

A prevalência de $\mathrm{IH}$ como a proporção da população que tem o evento ou doença de interesse em um determinado momento ou período de tempo específico também aponta algumas fontes que podem influenciar a taxa de prevalência de $\mathrm{IH}$ numa instituição. Esta pode aumentar com a maior duração da doença, com o aumento da sobrevida, com o aumento da incidência, com a imigração de casos, com a emigração de indivíduos sadios e com a mudança do método diagnóstico. Por outro lado, podem diminuir com a menor duração da doença, com a alta letalidade da doença, com a diminuição da incidência, com a imigração de indivíduos sadios e com a emigração de $\operatorname{casos}^{(5)}$.

A identificação da taxa de prevalência de $\mathrm{IH}$ depende da utilização de técnicas de Vigilância Epidemiológica, dos critérios de diagnóstico e dos fatores de risco intrínsecos e extrínsecos presentes numa determinada unidade em um dado tempo(5).

As unidades de terapia intensiva são de especial importância para prover dois serviços principais aos pacientes criticamente enfermos: suporte de vida para falências orgânicas graves e a monitorização intensiva que permita a identificação precoce e o tratamento apropriado das intercorrências clinicas graves. Constituem niveis de atendimento à saúde de alta complexidade, atuando de forma decisiva quando há instabilidade de órgãos e sistemas funcionais com risco de morte ${ }^{(6)}$.

Dessa forma, os pacientes admitidos em Unidade de Terapia Intensiva (UTI), estão sujeitos a riscos de 5 a 10 vezes maior de adquirir infecção que aqueles de outras unidades de internação do hospital, além de mais vulneráveis intrinsecamente à infecção, são freqüentemente expostos aos fatores de risco tais como: procedimentos invasivos, cirurgias complexas, drogas imunossupressoras, antimicrobianos e as interações com a equipe de saúde e os fômites ${ }^{(5)}$.

Diante dessa problemática, dos aspectos abordados e da vulnerabilidade dos pacientes internados na UTI, considerada área crítica, onde há um maior número de pacientes graves e submetidos à diversos procedimentos invasivos e portanto um maior numero de infecção(8), o estudo tem como objetivo determinar a prevalência de infecção hospitalar $(\mathrm{IH})$ e distribuição por topografia e por microorganismo e suas sensibilidades antimicrobianas.

\section{METODOLOGIA}

Trata-se de um estudo descritivo, retrospectivo, de abordagem quantitativa, realizado em um hospital público e de ensino, localizado na cidade de Teresina, no Estado do Piauí.

A população do estudo foi constituída por 647 pacientes que foram internados na UTI Geral e na UTI do Serviço de Pronto Socorro (SPS), ambas de atendimento à pacientes adultos, no período de janeiro a dezembro do ano de 2006. A amostragem foi constituída por 394 pacientes que desenvolveram infecção hospitalar no mesmo período. As UTI'S do HGV possuem 13 leitos, divididos em 6 leitos na UTI do SPS e 7 leitos na UTI geral. Na sua maioria, internam pacientes com poli-traumatismos, traumatismo crânio-encefálico (TCE), acidentes vasculares cerebrais (AVC's) provenientes do SPS e de outras clinicas como: médica, nefrologia, neurológica, ortopédica, cirúrgica, ginecológica, urológica e pneumológica.

Os dados foram coletados por meio da verificação de documentos referentes aos indicadores de infecção hospitalar, das culturas realizadas no ano de 2006, constante no banco de dados da Comissão de Controle de Infecção Hospitalar (CCIH) do hospital. O instrumento de coleta de dados foi um formulário estruturado com a identificação do serviço, o número da cultura, topografia da IH, tipos de microorganismos e sensibilidade aos antibióticos.

Para a análise dos dados foi utilizado o software SPSS, versão 9.0. Na análise estatística foram utilizadas medidas simples como: distribuição de freqüências e percentuais. Porém, para estudar a associação entre a infecção hospitalar e os tipos de procedimentos, foram calculadas as razões de prevalência. Os dados mais significativos foram apresentados em tabelas.

\section{APRESENTAÇÃO E ANÁLISE DOS RESULTADOS}

Os resultados do estudo de prevalência de $\mathrm{IH}$ realizado em um hospital público e de ensino, caracterizam a qualidade da estrutura física, organizacional e funcional do Hospital, com sérias complicações no processo de cuidar executado pelos profissionais da saúde, com destaque para os de Enfermagem, os quais dependem desses fatores, face à oferta da assistência requerida pelo cliente.

Verifica-se que o problema da infecção hospitalar, nas UTIs estudadas, apresenta-se grave e faz transformar as taxas encontradas em uma medida indireta da qualidade da assistência que está se prestando ao usuário deste serviço de saúde.

A prevalência de IH nas duas UTIs foi de $60,8 \%$. A UTI Geral foi responsável pelo maior índice de infecção, $64 \%$, enquanto a UTI do Pronto Socorro teve uma prevalência de $36 \%$.

A tabela 1 representa a prevalência de IH por topografia na UTI Geral e na UTI do Serviço de Pronto Socorro. As infecções mais freqüentes neste setor são a respiratória, sistêmica e a infecção de trato urinário, considerando que a maioria dos clientes faz uso de ventiladores mecânicos e cateter vesical. 
Tabela 1 Topografia das infecções por UTI. Teresina(PI), 2006.

\begin{tabular}{|c|c|c|c|c|c|c|}
\hline \multirow{3}{*}{ Topografia } & \multicolumn{4}{|c|}{ Tipo (Setor) } & \multirow{2}{*}{\multicolumn{2}{|c|}{ Total }} \\
\hline & \multicolumn{2}{|c|}{ UTI Geral } & \multicolumn{2}{|c|}{ UTI SPS } & & \\
\hline & $\mathrm{n}$ & $\%$ & $\mathrm{n}$ & $\%$ & $\mathrm{n}$ & $\%$ \\
\hline Respiratória & 155 & 61,26 & 82 & 58,16 & 237 & 60,15 \\
\hline Urinária & 44 & 17,39 & 20 & 14,18 & 64 & 16,24 \\
\hline Sistêmica & 39 & 15,42 & 31 & 21,99 & 70 & 17,77 \\
\hline Tegumentar & 8 & 3,16 & 3 & 2,13 & 11 & 2,79 \\
\hline Ferida Operatória & 7 & 2,77 & 5 & 3,55 & 12 & 3,05 \\
\hline Total & 253 & 100,00 & 141 & 100,00 & 394 & 100,00 \\
\hline
\end{tabular}

Fonte: CCIH do Hospital Getúlio Vargas, HGV

Fichas de protocolos de colheita de culturas. P> 0,05

Tabela 2. Tipos de microorganismos por UTI. Teresina(PI), 2006.

\begin{tabular}{|c|c|c|c|c|c|c|}
\hline \multirow{3}{*}{ Microorganismos isolados } & \multicolumn{4}{|c|}{ Tipo (Setor) } & \multirow{2}{*}{\multicolumn{2}{|c|}{ Total }} \\
\hline & \multicolumn{2}{|c|}{ UTI Geral } & \multicolumn{2}{|c|}{ UTI SPS } & & \\
\hline & $\mathrm{n}$ & $\%$ & $\mathrm{n}$ & $\%$ & $\mathrm{n}$ & $\%$ \\
\hline S. aureus & 35 & 13,94 & 32 & 22,70 & 67 & 17,09 \\
\hline Bgn (não fermentado) & 46 & 18,33 & 30 & 21,28 & 76 & 19,39 \\
\hline Pseudomonas aeruginosa & 14 & 5,58 & 5 & 3,55 & 19 & 4,85 \\
\hline Pseudomonas sp & 61 & 24,30 & 33 & 23,40 & 94 & 23,98 \\
\hline Escherichia coli & 8 & 3,19 & 9 & 6,38 & 17 & 4,34 \\
\hline Preoteus mirabilis & 1 & 0,40 & - & - & 1 & 0,26 \\
\hline Proteus sp & 1 & 0,40 & 1 & 0,71 & 2 & 0,51 \\
\hline Stafilococus epidermidis & 10 & 3,98 & 1 & 0,71 & 11 & 2,81 \\
\hline Klebisiela penumoniae & 89 & 35,46 & 43 & 30,50 & 132 & 33,67 \\
\hline Total & 251 & 100,00 & 141 & 100,00 & 392 & 100,00 \\
\hline
\end{tabular}

Fonte: CCIH do Hospital Getúlio Vargas, HGV

Fichas de protocolos de colheita de culturas.

\# Não somar 100\%, pode ser detectado mais de um microorganismo.

Tabela 3. Sensibilidade dos microorganismos de acordo com antibiograma. Teresina (PI), 2006.

\begin{tabular}{|c|c|c|c|c|c|c|}
\hline \multirow{3}{*}{ Sensibilidade antibiótica } & \multicolumn{4}{|c|}{ Tipo (Setor) } & \multirow{2}{*}{\multicolumn{2}{|c|}{ Total }} \\
\hline & \multicolumn{2}{|c|}{ UTI Geral } & \multicolumn{2}{|c|}{ UTI SPS } & & \\
\hline & $\mathrm{n}$ & $\%$ & $\mathbf{n}$ & $\%$ & $\mathrm{n}$ & $\%$ \\
\hline Amicacina & 120 & 47,06 & 78 & 52,48 & 194 & 48,99 \\
\hline Ampicilina & 29 & 11,37 & 18 & 12,77 & 47 & 11,87 \\
\hline Aztreonam & 120 & 47,06 & 61 & 43,26 & 181 & 45,71 \\
\hline Cefalexina & 30 & 11,76 & 25 & 17,73 & 55 & 13,89 \\
\hline Cefalotina & 28 & 10,98 & 19 & 13,48 & 47 & 11,87 \\
\hline Cefepime & 100 & 39,22 & 41 & 29,08 & 141 & 35,61 \\
\hline Cefotaxima & 66 & 25,88 & 31 & 21,99 & 97 & 24,49 \\
\hline Ceftazdima & 94 & 36,86 & 57 & 40,43 & 151 & 38,13 \\
\hline Ceftriaxona & 77 & 30,20 & 37 & 26,24 & 114 & 28,49 \\
\hline Ciprofloxacin & 95 & 37,25 & 56 & 39,72 & 151 & 38,13 \\
\hline Cefzolina & 29 & 11,37 & 24 & 17,02 & 53 & 13,38 \\
\hline Clindamicina & 20 & 7,84 & 24 & 17,02 & 44 & 11,11 \\
\hline Cloranfenicol & 53 & 20,78 & 40 & 28,37 & 93 & 23,48 \\
\hline Gentamicina & 86 & 33,73 & 50 & 35,06 & 136 & 34,34 \\
\hline Imipenem & 115 & 45,10 & 72 & 51,06 & 187 & 47,22 \\
\hline Oxacilina & 9 & 3,53 & 14 & 9,93 & 23 & 5,81 \\
\hline Perflexacin & 59 & 23,14 & 28 & 19,86 & 87 & 21,97 \\
\hline Penicilina & 11 & 4,31 & 6 & 4,26 & 17 & 4,29 \\
\hline Sulfametoxazol & 40 & 15,69 & 22 & 15,60 & 62 & 15,66 \\
\hline Vancomicina & 40 & 15,69 & 31 & 21,99 & 71 & 17,93 \\
\hline Sem registro & 4 & 1,57 & 1 & 0,71 & 5 & 1,26 \\
\hline
\end{tabular}

Fonte: CCIH do Hospital Getúlio Vargas, HGV

Fichas de protocolos de colheita de culturas.

\# Não somar $100 \%$, pode ser detectado mais de um tipo de sensibilidade. 
Nesta tabela, evidenciou-se a superioridade dos casos de infecção respiratória, especialmente na UTI geral, com $(61,26 \%)$ dos casos, seguida da UTI do Serviço de Pronto Socorro, com 58,16\%. Ainfecção sistêmica foi a segunda mais prevalente, com um total de 17,77 , seguida da urinária, com $16,24 \%$, nas duas UTIs. As topografias que apresentaram menor prevalência de infecção foram a tegumentar e a de ferida operatória. Assim, percebe-se que não há associação topográica entre as duas unidades de terapia intensiva.

As taxas observadas corroboram com a literatura, pois autores, com base em outros trabalhos relacionados com IH em UTIs, colocam que a infecção respiratória nas UTIs, apresenta-se como a segunda mais comum infecção nosocomial e com alta letalidade, variando entre 33\% a $71 \%$ e com relação entre caso e fatalidade podendo atingir até $55 \%$. Os casos de $86 \%$ de infecções respiratórias estão associados à pneumonia em pacientes em ventilação mecânica, sendo a infecção mais comum ${ }^{(8)}$.

As taxas de incidência de infecção hospitalar para pacientes internados em UTI variam, conforme o tipo de unidade e a população atendida, em razão da gravidade da doença básica, da restrição de pacientes no leito, do uso freqüente de sedação e das alterações no nível de consciência e dos múltiplos procedimentos invasivos das vias respiratórias, em que o principal fator de risco é o uso de ventilação mecânica associado ao tempo prolongado de utilização, equacionando também como fatores de risco: contaminação dos equipamentos e das soluções utilizadas na terapia ventilatória; condições favoráveis de aspiração, entre outros ${ }^{(5)}$

Em estudo de prevalência e multicêntrico, realizado em 22 hospitais da Turquia em 2001, foram analisados 56 UTIs, com 236 casos de $\mathrm{IH}$ relatados. Um total de 115 pacientes (48,7\%) apresentou um ou mais episódios de infecção nososcomias. Os sítios mais freqüentes foram a pneumonia no trato respiratório inferior (28\%), infecção de corrente sanguínea (23,3\%) e infecção urinária $(15,7 \%)$. Os fatores de risco associados podem ser o uso de tubo orotraqueal , presença de sonda vesical, traumas múltiplos, profilaxia de úlcera de estresse, presença de sonda nasogástrica, uso de ventilador mecânico(6).

Ainfecção de trato urinário (ITU) é uma das mais freqüentes na população adulta. Cerca de $70 \%$ a $88 \%$ dos casos de ITU ocorrem em pacientes submetidos a cateterismo vesical por ser um procedimento invasivo. A duração do cateterismo é um dos fatores que contribuem para a ocorrência da infecção urinária. A ocorrência da infecção depende das características do microorganismo causador, do tamanho do seu inoculo e da defesa do hospedeiro. AE. coli é o principal agente isolado nas bacteriúrias hospitalares. Os microorganismos, pseudomonas aeruginosas, klebisiela pneumoniae, proteus sp e o enterococo estão entre os agentes mais comuns ${ }^{(7)}$.

Segundo dados epidemiológicos, $35 \%$ a $45 \%$ de todos os casos de infecções hospitalares adquiridas, são infecções do trato urinário, sendo que $80 \%$ estão relacionadas ao uso de cateter vesical de demora (principal veiculo de transmissão)(9)

A tabela 2 representa a prevalência de $I H$ por tipo de microorganismos na UTI Geral e na UTI do Serviço de Pronto Socorro.

Nesta tabela, evidencia-se que o microorganismo causador do maior número de infecções foi a Klebsiella Pneumoniae (35,46\%), na UTI geral, seguida da UTI do SPS, com $30,50 \%$ dos casos. Os Pseudomonas spp foi o segundo microorganismo mais prevalente, com $24,30 \%$, na UTI geral, seguida da UTI do SPS, com $23,40 \%$. Os microorganismos que apresentaram menor prevalência nos resultados das culturas foram Proteus spp e Proteus mirabilis.

Estudos evidenciam uma taxa de infecção alta entre pacientes de terapia intensiva, especialmente nas infecções respiratórias cujas bactérias predominantes foram: Klebsiella pneumoniae, Pseudomonas aeruginosa e Staphylococcus aureus (agentes muitiresistentes). Outro estudo desenvolvido na Turquia com 56 UTIs, demonstrou a prevalência desses mesmos microorganismos, Pseudomonas aeruginosa(20,8\%), Staphylococcus aureus $(18,2 \%)$, Klebsiella pneumoniae $(16,1 \%)^{(6)}$.

Pesquisa de prevalência realizada em São Paulo, no ano de 2003, com duração de somente um dia, envolvendo 19 UTIs, identificou 126 casos de $\mathrm{IH}, 87$ pacientes receberam antimicrobianos no dia do estudo, $72(57 \%)$ para tratamento e $15(12 \%)$ para profilaxia. Neste estudo os agentes mais freqüentemente isolados foram: Enterobacteriaceae (33,8\%), pseudomonas aeruginosa $(26,4 \%)$ e Staphylococcus aureus (16,9\%; 100\% MRSA) Staphylococcus aureus meticilina resistente. Esta análise multivariada identificou como fatores de risco associados à infecção adquirida em UTI: idade maior ou igual a 60 anos, uso de sonda nasogástrica e pós-operatório(6).

Fatores como o uso de sonda nasogástrica, pós-operatório, idade maior ou igual a 60 anos, procedimentos invasivos, cirurgias complexas, drogas imunossupressoras, sedação e as interações com a equipe de saúde e os fômites, mostraram associação com infecção( ${ }^{(5)}$.

A tabela 3 representa a sensibilidade dos microorganismos aos antibióticos utilizados na UTI Geral e na UTI do Serviço de Pronto Socorro. TABELA 3

Na tabela 3, evidenciou-se nos antibiogramas, uma maior sensibilidade bacteriana ao aminoglicosideo amicacina, com $48,99 \%$, seguido do imipenem, com $47,22 \%$ e do aztreonan com $45,71 \%$, nas duas UTI's.

Os aminoglicosídeos (amicacina, streptominina, gentamicina, neomicina e tobramicina) são antibióticos bactericidas com rápida ação letal para os bacilos aeróbicos gram-negativos sensíveis ${ }^{(10)}$. 0 uso da amicacina é recomendado apenas no tratamento de infecções graves causadas por bactérias sensíveis nos casos de sepse, pneumonia e outras infecções graves de comprometimento do estado geral do paciente, na maioria das vezes em associação com outros antimicrobianos ${ }^{(11)}$.

O imipenem, a exemplo de outros antibióticos Beta-Lactâmico, liga-se às proteínas de ligação da penicilina, interrompe a síntese da parede celular bacteriana e provoca morte dos microorganismos sensíveis. Sua atividade in vitro é excelente para uma ampla variedade de microorganismos aeróbicos e anaeróbicos. Os streptococos, os enterococos e as cepas resistentes à penicilina não-produtora de Beta- Lactamase, os stafilococos (incluindo cepas produtoras de penicilase), são todas sensiveis. É indicado para o tratamento de infecções do trato urinário, das vias respiratórias inferiores, infecções intra-abdominais e ginecológicas, cutâneas, dos tecidos moles, dos ossos e das articulações ${ }^{(12)}$

$O$ aztreonan, também é um composto Beta-Lactâmico que se mostra resistente a muitas das Beta-Lactamases elaboradas pela maioria das bactérias gram-negativas. A terapia com esse antimicrobiano Beta- Lactâmicos é dinâmico. A prevalência da resistência bacteriana a esse antimicrobiano continua aumentando nos serviços de saúde, enquanto outros novos e mais eficazes são desenvolvidos para uso clinico ${ }^{(12)}$.

$O$ advento dos antibióticos e quimioterápicos permitiram o controle e cura das doenças infecciosas, mudando a evolução natural dessas doenças de forma marcante. Porém, dez anos depois de descoberta a penicilina e antes mesmo de estar disponível para uso clínico, foi identificada a presença de beta-lactamases em bactérias, caracterizando resistência de algumas espécies e logo o surgimento de resistência adquirida aos antimicrobianos passou a ser um problema cada vez mais preocupante ${ }^{(13)}$.

Dessa forma, os antibióticos representam um item de alto consumo em hospitais, em particular em unidades de pacientes mais graves, como as UTIs. Ainadequação e o uso abusivo de antibióticos e a falta de critérios na escolha do tratamento empírico, são fatores determinantes para o surgimento de diversos microorganismos resistentes em uma unidade de terapia intensiva ${ }^{(5)}$.

A tabela 4 representa a sensibilidade dos três microorganismos mais prevalentes no estudo, aos antibióticos utilizados na UTI Geral e na UTI do Serviço de Pronto Socorro.

Evidencia-se na tabela 4 a predominância da sensibilidade do microrganismo S. aureus a vancomicina(100\%), a pseudomonas spp a aztreonam $(64,77 \%)$ e a Klebsiella pneumoniae ao antimicrobiano imipenem $(62,2 \%)$

A vancomicina é um glicopeptídeo, atualmente em uso clínico, que tem seu uso restrito ao tratamento das infecções por gram-positivos ${ }^{(9)}$. Porém, 
Tabela 4. Tipo de microorganismo por sensibilidade. Teresina(PI), 2006.

\begin{tabular}{|c|c|c|c|c|c|c|c|c|}
\hline \multirow{3}{*}{ Sensibilidade antibiótica } & \multicolumn{6}{|c|}{ Tipo de Microorganismo } & \multirow{2}{*}{\multicolumn{2}{|c|}{ Total }} \\
\hline & \multicolumn{2}{|c|}{ S. aureus } & \multicolumn{2}{|c|}{$\begin{array}{c}\text { Pseudomonas } \\
\text { aeruginosa }\end{array}$} & \multicolumn{2}{|c|}{ Klebisiela penumoniae } & & \\
\hline & $\mathrm{n}$ & $\%$ & $\mathrm{n}$ & $\%$ & $\mathrm{n}$ & $\%$ & $\mathrm{n}$ & $\%$ \\
\hline Amicacina & 40 & 67,80 & 40 & 45,45 & 57 & 44,88 & 137 & 50,00 \\
\hline Ampicilina & 16 & 27,12 & 3 & 3,41 & 15 & 11,81 & 34 & 12,41 \\
\hline Aztreonam & 7 & 11,86 & 57 & 64,77 & 65 & 51,18 & 129 & 47,08 \\
\hline Cefalexina & 29 & 49,15 & 2 & 2,27 & 12 & 9,45 & 43 & 15,69 \\
\hline Cefalotina & 25 & 42,37 & 2 & 2,27 & 8 & 6,30 & 35 & 12,77 \\
\hline Cefepime & 7 & 11,86 & 33 & 37,50 & 60 & 47,24 & 100 & 36,50 \\
\hline Cefotaxima & 31 & 52,54 & 13 & 14,77 & 33 & 25,98 & 77 & 28,10 \\
\hline Ceftazdima & 22 & 37,29 & 35 & 39,77 & 52 & 40,94 & 109 & 39,78 \\
\hline Ceftriaxona & 32 & 54,24 & 19 & 21,59 & 36 & 28,35 & 87 & 31,75 \\
\hline Ciprofloxacin & 33 & 55,93 & 34 & 38,64 & 45 & 35,43 & 112 & 40,88 \\
\hline Cefzolina & 27 & 45,76 & 1 & 1,14 & 11 & 8,66 & 39 & 14,23 \\
\hline Clindamicina & 29 & 49,15 & 4 & 4,55 & 4 & 3,15 & 37 & 13,50 \\
\hline Cloranfenicol & 37 & 62,71 & 6 & 6,82 & 23 & 18,11 & 66 & 24,09 \\
\hline Gentamicina & 34 & 57,63 & 30 & 34,09 & 38 & 29,92 & 102 & 37,23 \\
\hline Imipenem & 8 & 13,56 & 38 & 43,18 & 79 & 62,20 & 125 & 45,62 \\
\hline Oxacilina & 20 & 33,90 & 1 & 1,14 & - & - & 21 & 7,66 \\
\hline Perflexacin & 3 & 5,08 & 24 & 27,27 & 31 & 24,41 & 58 & 21,17 \\
\hline Penicilina & 9 & 15,25 & 2 & 2,27 & 2 & 1,57 & 13 & 4,74 \\
\hline Sulfametoxazol & 29 & 49,15 & 4 & 4,55 & 19 & 14,96 & 52 & 18,98 \\
\hline Vancomicina & 55 & 93,22 & 2 & 2,27 & 3 & 2,36 & 60 & 21,90 \\
\hline Sem registro & - & - & 1 & 1,44 & 1 & 0,79 & 2 & 0,73 \\
\hline
\end{tabular}

Fonte: $\mathrm{CClH}$ de um Hospital de ensino.

\# Não somar 100\%, um microorganismo pode ter mais de uma sensibilidade.

nos últimos anos, o aumento das infecções por patógenos gram-positivos foram destaque em diferentes publicações ${ }^{(14)}$.

O S. aureus é considerado um patógeno humano oportunista e com freqüência está associado às infecções hospitalares. De acordo com o National Nosocomial Infections Surveillande do Center for Disease Control and Prenventio (CDC), desde 1999, a proporção de S. aureus resistente à meticilina (Staphylococcus aureus meticilina-resistentes - MRSA) ultrapassa $50 \%$ entre os pacientes internados em UTI ${ }^{(21)}$.

No Brasil, as infecções hospitalares causadas por $\mathrm{S}$. aureus resistentes à meticilina, tambémé elevado, correspondendo de $40 \%$ a $80 \%$, pricipalmente nas UTIs. Pesquisas recentes demonstraram altos índices de mortalidade em pacientes que desenvolveram bacteremia por MRSA, $49 \%$ a $55 \%$, do que por $\mathrm{S}$. aureus sensível à meticilina (MSSA), $20 \%$ a $32 \%\left({ }^{(14)}\right.$.

A Pseudomona é um gênero de bacilos gram-negativos aeróbios da família pseudomoneacea. São patógenos para planta e somente algumas espécies estão associadas com doenças no ser humano. A sua patogênese deve ser discutida no contexto de uma infecção oportunista, sendo necessária a existência de quebra de barreiras ou de defeitos específicos de alguns dos mecanismos de defesa imune e em contato com o homem, pode colonizar diversos tecidos. As manifestações clínicas mais prevalentes são: pneumonias, infecções de cateter vascular central, infecção de trato uninário, dentre outras ${ }^{(15)}$.

0 aztreonan é um antibacteriano beta-lactâmico monocíclico com indicações para infecções respiratórias causadas por: klebisiella pneunoniae, pseudomonas spp, proteus mirabilis e escherichia coli(16,17).

A Klebisiela pneumoniae é um bacilo gram-negativo presente no trato gastrointestinal de indivíduos colonizados e é um importante patógeno causador de infecções hospitalares, especialmente em unidades de tratamento crítico como nas UTIs.

A resistência da Klebisiela pneumoniae produtora de beta-lactamase de espectro ampliado (ESBL), classe de enzimas que conferem resistência a todas as cefalosporina, tem causado preocupação nos serviços de terapia intensiva. Porém, a maioria das Klebisielas pneumoniae, apresenta a beta- lactamase SHV-1. A Klebisiela pneumoniae oxytoca, apresenta a enzima tipo K1. No entanto, a enzima K1 possue uma atividade mais ampla em relação à SHV1, e 20\% das Klebisielas pneumoniae oxytoca, mutantes, hiperproduzem essa bata-lactamese e são resistente à todas as penicilinas, cefotaxina, cefetriaxon, astreonan, mas não são resistentes aos carbapênicos (imipenem), pois apresentam maior estabilidade ${ }^{(14)}$.

Dessa forma, os microorganismos $S$. aureus, pseudomonas spp e a Klebsiella pneumoniae fazem parte da flora microbiana das duas unidades de terapia intensiva estudadas, demonstrando sensibilidade aos antimicrobianos padronizados e alguns de uso restrito, necessitando de vigilância por parte da $\mathrm{CCIH}$ do hospital.

\section{CONCLUSÃO}

A taxa de prevalência de IH nas duas UTIs foi de 60,8\%. AUTI Geral foi responsável pelo maior índice de infecção $64 \%$, enquanto a UTI do Pronto Socorro teve uma prevalência de $36 \%$. Evidenciou-se que o microorganismo causador do maior número de infecções foi a Klebsiella Pneumoniae (35,46\%) na UTI geral, seguida da UTI do SPS, com $30,50 \%$ dos casos. 0 Pseudomona spp foi o segundo microorganismo mais prevalente, com $24,30 \%$ na UTI geral, seguido da UTI do SPS, com $23,40 \%$. Os microorganismos que apresentaram menor prevalência nos resultados das culturas foram Proteus spp e Proteus mirabilis.

Nos antibiogramas, percebeu-se uma maior sensibilidade bacteriana ao aminoglicosídeo amicacina, com 48,99\%, seguido do imipenem, com 47,22\% e do aztreonan, com $45,71 \%$, nas duas UTI's. A predominância da sensibilidade do microrganismo S. aureus foi a vancomicina (100\%), da pseudomonas spp foi a aztreonam (64,77\%) e a Klebsiella pneumoniae ao antimicrobiano imipenem (62,2\%).

Nas UTIs, os fatores propícios para o desenvolvimento de uma IH como: o tempo de permanência prolongado, o uso de ventilação mecânica e procedimentos invasivos, a susceptibilidade dos pacientes, idade, uso de imunossupressores, doenças de base e condições nutricionais, têm contribuído 
para a prevalência de infecções neste serviço, necessitando de uma vigilância permanente por parte da Comissão de Controle de Infecção Hospitalar $(\mathrm{CClH})$ do hospital, conforme preconizado pelo Programa Nacional de Controle de Infecção Hospitalar do MS.

Dessa forma, o MS estabelece que $70 \%$ das IH são controláveis e 30\% são preveníveis. Considerando esses dados, nas UTIs do hospital estudado, $18,24 \%$ de infecções poderiam ser preveníveis se o referido Programa de Controle de $\mathrm{lH}$ fosse bem desenvolvido e precauções fossem tomadas para evitar as infecções cruzadas, transmitidas, provavelmente, pelas mãos dos profissionais da saúde, por não usarem a técnica correta da lavagem das mãos, caracterizada, isoladamente, como a ação mais importante para a prevenção e controle das $\mid \mathrm{H}$.

Além disso, a vigilância epidemiológica, precaução padrão, medidas de isolamento, materiais e equipamentos adequados, higienização do ambiente, identificação de bactérias multi-resistente, antibioticoterapia adequada, treinamento da equipe multiprofissional, implementação de medidas de controle, são fatores importantes e determinantes que podem interferir nos resultados com redução das taxas de prevalência de infecção hospitalar.

\section{REFERÊNCIAS}

1. Ministério da Saúde (BR). Expede na forma de anexos diretriz e normas para a prevenção e controle das infecções hospitalares: Portaria No 2.616, de 12 de maio de 1998. Diário Oficial da União, República Federativa do Brasil, Brasília (DF), jul 1998.

2. Prade SS. Estudo Brasileiro da Magnitude das Infecões Hospitalares em Hospitais Terciários. Rev Controle Infecão Hosp 1995;2(2).

3. Moura MEB. Infecção hospitalar no Piauí: a crítica e os aspectos críticos no processo de cuidar/cuidado (tese). Rio de Janeiro(RJ): Escola de Enfermagem Anna Nery, Universidade Federal do Rio de Janeiro; 2001.

4. Senado Federal (BR). Constituição: República Federativa do Brasil: 1988. Brasília (DF): Centro Gráfico; 1988

5. Couto RC, Pedrosa TMG, Nogueira JM. Infecão Hospitalar Epidemiologia, Controle e tratamento. $3^{\text {a }}$ ed. Rio de Janeiro, Editora Médica e Científica; 2003.

6. Martins P. Epidemiologia das Infecções em centro de terapia intensiva de adulto (tese). Belo Horizonte (MG): Universidade de Minas Gerais; 2006.

7. Gagliarbi EMDB, Fernandes AT, Cavalcante NJF. Infecção do trato urinário. In: Fernandes AT, Fernandes MOV, Ribeiro Filho N. Infecção hospitalar e suas interfaces na área da saúde. $1^{\mathrm{a}}$ ed. São Paulo(SP): Atheneu; 2000. p. 459-78.

8. Pedrosa TMG, Macedo R.M. Limpeza hospitalar. In: Couto RC Pedrosa TMG. Guia prático de infecção hospitalar. Rio de Janeiro (RJ): Medsi; 1999.

9. Guimarães MMQ, Rocco JR. Prevalência e prognóstico dos pacientes com pneumonia associadas a ventilação mecânica em um hospital universitário. Rev Bras Pneumol 2006;22(4):33946.

10. Sampaio JRN. Mecanismo de ação dos antibióticos. In: Neto VA, Nicodemo AC, Lopes HV. Antibiótico na prática médica. $6^{\mathrm{a}}$ ed. São Paulo (SP): Sarvier; 2007. p. 17-25.

11. Penteado Filho SR, Lopes HV, Levi GC. Antibióticos clássicos: principais características e uso terapêutico. In: Neto VA, Nicodemo AC, Lopes HV. Antibiótico na prática médica. $6^{a}$ ed. São Paulo (SP): Sarvier; 2007. p. 87-152.

12. Petre Jr WA. Antimicrobianos: penicilinas, cefalosporinas e outros antibióticos beta-lactâmicos. In: Goodman A. As bases farmacológicas da terapêutica. $10^{\mathrm{a}}$ ed. Rio de Janeiro (RJ): McGraw-Hill; 2003. p. 891-912.

13. Moreira LB. Princípios para o uso de Antimicrobianos. Rev AMRIGS 2004;48(2):73-152.

14. Rossi F, Andreazze DB. Resistência Bacteriana - interpretando 0 antibiograma. $1^{a}$ ed. São Paulo (SP): Atheneu; 2005.

15. Levin ASS, Arruda EAG, Oliveira MS. Infecções por pseudomonas. In: Veronese R, Focaccia R. Tratado de infectologia. $3^{a}$ ed. São Paulo (SP): Atheneu; 2005. p. 971-79.

16. Zanini AC, Basile AC, Folador W, Oga S. Guia de medicamentos. $2^{a}$ ed. São Paulo (SP): Ipex Comercial Ltda; 1998.

17. Biamcalana ML. Terapêutica antimicrobiana das principais síndromes e doenças infecciosas. In: Neto VA, Nicodemo AC, Lopes HV. Antibióticos na prática médica. $6^{a}$ ed. São Paulo (SP): Sarvier; 2007. 